\title{
ON THE GROWTH OF THE TAYLOR COEFFICIENTS OF AUTOMORPHIC FORMS
}

\author{
THOMAS A. METZGER ${ }^{1}$
}

\begin{abstract}
The growth of the Taylor coefficients of an automorphic form of dimension -2 with respect to a Fuchsian group $\Gamma$ is related to the area integral $\iint_{U}|F|^{s}\left(1-|z|^{2}\right)^{t} d x d y$, and it is found that these coefficients must grow faster than a power of $n$. Moreover if $F \in H(p, \Gamma)$ then these coefficients must grow slower than a different power of $n$ and, in fact, $a_{n} / n$ is square summable if either $p=2$ or $1<p<\infty$ and $\Gamma$ is finitely generated of the second kind.
\end{abstract}

1. Introduction. Throughout $\Gamma$ shall stand for a Fuchsian group acting on the unit disk $U(=\{z:|z|<1\})$ of the complex plane. We shall assume that $\Gamma$ is of convergence type, i.e.,

$$
\sum_{T \in \Gamma}\left|T^{\prime}(z)\right|<\infty \text { for all } z \text { in } U .
$$

We note that (1.1) is equivalent to the fact that the associated Riemann surface $U / \Gamma$ is hyperbolic (cf. Tsuji $[9$, p. 522]). If $F$ is a function defined on $U$, then we say that $F$ is an automorphic form of degree -2 if

$$
F(T z) T^{\prime}(z)=F(z) \text { for all } T \text { in } \Gamma \text { and } z \text { in } U \text {. }
$$

We define

$$
\lambda^{-1}(z)=\left(1-|z|^{2}\right), \quad d \omega(z)=\lambda^{2}(z) d x d y,
$$

and note that $\lambda^{-1}(T z)=\left|T^{\prime}(z)\right| \lambda^{-1}(z)$ and $d \omega(T z)=d \omega(z)$ for all conformal automorphisms $T$ of $U$ onto itself. If $\Omega$ is a fundamental region for $\Gamma$ whose boundary has two dimensional measure zero, then we define $H(p, \Gamma)(1 \leqq p<\infty)$ to be the space of holomorphic automorphic forms of

Presented to the Society, January 17, 1972 under the title On the Taylor coefficients of automorphic forms; received by the editors March 16, 1972 and, in revised form, September 29, 1972.

AMS (MOS) subject classifications (1970). Primary 30A58; Secondary 30A10.

Key words and phrases. Taylor coefficients, $p$-integrable, automorphic forms, Fuchsian groups.

${ }^{1}$ Part of the results are contained in the author's $1971 \mathrm{Ph} . \mathrm{D}$. dissertation at Purdue University, written under Professor K. V. Rajeswara Rao. Also the author wishes to thank the referee for his helpful comments on the original version.

(c) American Mathematical Society 1973 
degree -2 with respect to $\Gamma$ such that $\|F\|_{p}<\infty$ where

$$
\|F\|_{p}^{p}=\iint_{\Omega}|F(z)|^{p} \lambda^{-p}(z) d \omega(z) .
$$

We note that $H(1, \Gamma)=\{0\}$ for a large class of groups $\Gamma$ (cf. [5, Theorem 3]). $H(\infty, \Gamma)$ is defined to be the space of holomorphic automorphic forms of degree -2 with respect to $\Gamma$ with the norm

$$
\|F\|_{\infty}=\sup _{z \in U}|F(z)| \lambda^{-1}(z)<\infty .
$$

In this paper we shall obtain estimates on the growth of the Maclaurin coefficients of holomorphic automorphic forms of dimension -2 by relating such growth to the finiteness of integrals of the form (cf. [3])

$$
\iint_{U}|F|^{s}\left(1-|z|^{2}\right)^{t} d x d y .
$$

Although all of our work is done for the case $\Gamma$ of convergence type and automorphic forms of dimension -2 , analogous results could be stated and proved for arbitrary $\Gamma$ and automorphic forms of dimension $-2 q<-2$.

2. Lower limits on the order of growth of the coefficients. We shall first show that the Maclaurin coefficients of an automorphic form cannot grow "too" slowly.

THEOREM 1. Suppose $\Gamma$ is such that $\sum_{T \in \Gamma}\left|T^{\prime}(z)\right|^{r}=\infty$ for some $z$ in $U$. Let $F(z)=\sum_{n=0}^{\infty} a_{n} z^{n}, F \not \equiv 0$, be an arbitrary (holomorphic) automorphic form of degree -2 with respect to $\Gamma$, then, for any $t<r / 2, a_{n} \neq O\left(n^{t}\right)$.

Note. If $\sum_{T \in \Gamma}\left|T^{\prime}(t)\right|^{r}=\infty$ for one $z$ in $U$ then $\sum_{T \in \Gamma}\left|T^{\prime}(t)\right|^{r}=\infty$ for all $z$ in $U$.

Proof. Suppose $a_{n}=O\left(n^{t}\right)$, then $\sum_{n=0}^{\infty}\left(\left|a_{n}\right|^{2} /(n+1)^{1+r}\right)<\infty$. Since $\Gamma(x) / \Gamma(x+a) \sim x^{-a}$, it follows that

$$
\sum_{n=0}^{\infty} \frac{\left|a_{n}\right|^{2} \Gamma(r+1) \Gamma(n+1)}{\Gamma(n+r+2)}<\infty,
$$

this in turn implies, since $\beta(p, q)=\Gamma(p) \Gamma(q) / \Gamma(p+q)$,

$$
\sum_{n=0}^{\infty}\left|a_{n}\right|^{2} \int_{0}^{1}(1-u)^{r} u^{n} d u<\infty .
$$

Now upon letting $u=\rho^{2}$, where $|z|=\rho$, we see that Pareseval's formula 
implies that

$$
\iint_{U}|F|^{2}\left(1-|z|^{2}\right)^{r} d x d y=\sum_{n=0}^{\infty}\left|a_{n}\right|^{2} \int_{0}^{1}\left(1-\rho^{2}\right)^{r} \rho^{2 n+1} d \rho<\infty .
$$

But this cannot happen if $F \not \equiv$ for

$$
\begin{aligned}
\infty & >\iint_{U}|F|^{2}\left(1-|z|^{2}\right)^{r} d x d y=\sum_{T \in \Gamma} \iint_{T \Omega}|F|^{2}\left(1-|z|^{2}\right)^{r} d x d y \\
& =\iint_{\Omega}|F|^{2}\left(1-|z|^{2}\right)^{r} \sum_{T \in \Gamma}\left|T^{\prime}(z)\right|^{r} d x d y,
\end{aligned}
$$

since $|F|^{2} d x d y$ is $\Gamma$ invariant. Since $\sum_{T \in \Gamma}\left|T^{\prime}(z)\right|^{r} \equiv \infty$ we have arrived at a contradiction and the proof of the theorem is complete.

COROLlaRY 2. Suppose $F$ is as above and $\Gamma$ is not cyclic hyperbolic, then $a_{n} \neq 0(1)$.

Proof. If $\Gamma$ is not cyclic hyperbolic, then Beardon [1] proved that there exists an $r>0$ such that $\sum_{r \in \Gamma}\left|T^{\prime}(t)\right|^{r}=\infty$; hence choosing $t=0$ in Theorem 1 completes the proof.

REMARK. If $\Gamma$ is cyclic hyperbolic, Beardon $[1, \mathrm{p} .475]$ showed that $\sum_{T \in \Gamma}\left|T^{\prime}(t)\right|^{r}<\infty$ for all $r>0$, however in this case we have $a_{n} \neq O\left(n^{-\varepsilon}\right)$ for all $\varepsilon>0$.

3. Upper limits on the growth of the coefficients. We note that in the above theorem and its corollary no assumption was made about the growth of $F$. If we make such an assumption, i.e., $F \in H(p, \Gamma)$, then it can be shown that the Maclaurin coefficients of $F$ cannot grow "too" fast. In order to prove this we first need

Lemma 3. Let $F \in H(p, \Gamma)(1 \leqq p<\infty)$. Then (1.3) is finite with $s=p$ and $t=p-1$.

ProOF.

$$
\begin{aligned}
\iint_{U}|F|^{p}\left(1-|z|^{2}\right)^{p-1} d x d y & =\iint_{U}\left|F \lambda^{-1}\right|^{p}\left(1-|z|^{2}\right) d \omega(z) \\
& =\iint_{\Omega}\left|F \lambda^{-1}\right|^{p} \sum_{T \in \Gamma}\left(1-|T z|^{2}\right) d \omega(z) \\
& \leqq\left[\sup _{U} \sum_{T \in \Gamma}\left(1-|T z|^{2}\right)\right]\|F\|_{p}^{p} .
\end{aligned}
$$


This last term is finite by Theorem 3 of [7] and the fact $F \in H(p, \Gamma)$, if $\Gamma$ acts freely on $U$. If the origin is not a fixed point of $\Gamma$, then the proof of Theorem 3 of [7] goes through without modification to yield the desired result. If the origin is a fixed point of $\Gamma$, then, by conjugation, one can return to the previous case and again the result follows. This completes the proof of the lemma.

Proposition 4. Suppose $F(z)=\sum_{n=0}^{\infty} a_{n} z^{n} \in H(p, \Gamma)(1<p<\infty)$. Then (1.3) is finite if $s=1$ and $t>0$; thus $a_{n}=O\left(n^{1+t}\right)$ as $n \rightarrow \infty$.

Proof.

$$
\begin{aligned}
& \iint_{U}|F|\left(1-|z|^{2}\right)^{t} d x d y=\iint_{U}|F|\left(1-|z|^{2}\right)^{(p-1) / p}\left(1-|z|^{2}\right)^{t-(p-1) / p} d x d y \\
& \leqq\left(\iint_{U}|F|^{p}\left(1-|z|^{2}\right)^{p-1} d x d y\right)^{1 / p} . \\
&\left(\iint_{U}\left(1-|z|^{2}\right)^{t p /(p-1)-1} d x d y\right)^{(p-1) / p}<\infty,
\end{aligned}
$$

by Hölder's inequality, Lemma 3 , and the fact $t>0$. The conclusion that $a_{n}=O\left(n^{1+t}\right)$ as $n \rightarrow \infty$ now follows from Theorem 4 of [3].

REMARK. J. Lehner (private communication) has shown that if

$$
F \in H(p, \Gamma) \quad(1 \leqq p<\infty), \quad \text { then } a_{n}=O(n) .
$$

4. Summability results. From Proposition 4 we saw that $a_{n}=O\left(n^{1+t}\right)$ for all $t>0$ if $\sum_{n=0}^{\infty} a_{n} z^{n} \in H(p, \Gamma)$. However even more is true if we assume $2 \leqq p<\infty$ for arbitrary $\Gamma$ or $1<p<\infty$ and $\Gamma$ a finitely generated group. We note that if $\Gamma$ is finitely generated then $\Gamma$ is of convergence type if and only if $\Gamma$ is of the second kind. We first prove

LEMMA 5. If $\Gamma$ is finitely generated of the second kind then, for $1<p<\infty$,

$$
\iint_{\Omega}\left(\sum_{T \in \Gamma} 1-|T z|^{2}\right)^{p} d \omega(z)<\infty .
$$

The idea of proof is that if one chooses $\Omega$ to be a Dirichlet region, then, $\partial \Omega \cap \partial U$ consists of free sides and parabolic cusps. On the free sides $\sum_{T \in \Gamma}\left|T^{\prime}(z)\right|$ has a bounded supremum and so we must integrate $\left(1-|z|^{2}\right)^{p-2} d x d y$ which is finite. As for the parabolic cusps we need only recall that the hyperbolic area of a parabolic cusp is finite (cf. [4] for the details). Since $\|\cdot\|_{p}$ is independent of the choice made for the fundamental region the result follows. 
TheOREM 6. Suppose $F(z)=\sum_{n=0}^{\infty} a_{n} z^{n}$. Then $\left\{a_{n} / n\right\} \in l_{2}$ if either

(i) $F \in H(2, \Gamma)$ or

(ii) $F \in H(p, \Gamma)(1<p<\infty)$ and $\Gamma$ is finitely generated.

Proof. In view of Parseval's formula for functions holomorphic in $U$ it suffices to show that (1.3) is finite with $s=2$ and $t=1$. Lemma $3 \mathrm{im}$ mediately yields the result in case (i). If $\Gamma$ is finitely generated, then, for $1<p \leqq q \leqq \infty$,

$$
H(p, \Gamma) \subseteq H(q, \Gamma) .
$$

Assuming this for the moment, we see immediately that the theorem is true for $1<p \leqq 2$ in case (ii). If $p>2$, then

$$
\begin{aligned}
\iint_{U}|F|^{2}\left(1-|z|^{2}\right) d x d y= & \iint_{U}\left|F \lambda^{-1}\right|^{2}\left(1-|z|^{2}\right) d \omega(z) \\
= & \iint_{\Omega}\left|F \lambda^{-1}\right|^{2} \sum_{T \in \Gamma}\left(1-|T z|^{2}\right) d \omega(z) \\
\leqq & \left(\iint_{\Omega}\left|F \lambda^{-1}\right|^{p} d \omega(z)\right)^{2 / p} \\
& \times\left(\iint_{\Omega}\left(\sum_{T \in \Gamma}\left(1-|T z|^{2}\right)\right)^{p /(p-2)} d \omega(z)\right)^{(p-2) / p} \\
& <\infty,
\end{aligned}
$$

by Lemma 5 and the fact $F \in H(p, \Gamma)$ with $p>2$. Thus the proof will be complete once (4.2) is established. This shall be done in $\S 5$.

In case $\Gamma$ is infinitely generated of convergence type and $F \in H(p, \Gamma)$ $(p \neq 2)$, similar methods to those above yield then

Proposition 7. If $\Gamma$ is of convergence type and $F(z)=\sum_{n=0}^{\infty} a_{n} z^{n} \in H(p, \Gamma)$,

(i) $\left\{a_{n} n^{-r}\right\} \in l_{2}$ for all $r>1$ if $2<p<\infty$,

(ii) $\left\{a_{n} n^{-(p+2) / 2 p}\right\} \in l_{2}$ if $1<p \leqq 2$.

The idea of the proof is to show that (1.3) is finite if $s=2$ and $t>1$ in case (i) and $s=2$ and $t=2 / p$ in case (ii). Then using the fact $\beta(p, q)=$ $\Gamma(p) \Gamma(q) / \Gamma(p+q)$ and $\Gamma(x) / \Gamma(x+a) \sim x^{-a}$ the result follows by Parseval's formula.

5. An auxiliary result. In the proof of Theorem 6 we merely asserted (4.2). To prove this assertion we need to introduce the following notation analogous to that of [5]. Given $z, \zeta \in U$, we define

$$
k(z, \zeta)=\pi^{-1}(1-z \bar{\zeta})^{-2}
$$


and

$$
\begin{aligned}
\alpha(z, \zeta) & =\theta(k(z, \zeta), \Gamma) \\
& =\sum_{T \in \Gamma} k(T z, \zeta) T^{\prime}(z) .
\end{aligned}
$$

It is known (cf. [7, Theorem 1]) that (every arrangement of) the series defining $\alpha(z, \zeta)$ converges, for fixed $\zeta$ in $U$, uniformly on compact subsets of $U$. Moreover since $k(z, \zeta)$ is a bounded analytic function for each $\zeta$ in $U$ it follows that $\alpha(z, \zeta) \in H(p, \Gamma)(1<p \leqq \infty)$ whenever $\Gamma$ is finitely generated. Also we have

Proposition 8. Suppose $\Gamma$ is finitely generated of the second kind and $F \in H(p, \Gamma)(1<p<\infty)$. Then

$$
\iint_{\Omega} F(z) \overline{\alpha(z, \zeta)} d x d y=F(\zeta)
$$

Proof. Formally we have

$$
\begin{aligned}
\iint_{\Omega} F(z) \overline{\alpha(z, \zeta)} d x d y & =\iint_{\Omega} F(z) \sum_{T \in \Gamma} \overline{k(T z, \zeta)} \overline{T^{\prime}(z)} d x d y \\
& =\iint_{U} F(z) \overline{k(z, \zeta)} d x d y=F(\zeta) .
\end{aligned}
$$

To complete the proof it suffices to show that

$$
I=\iint_{U}|F(z)||k(z, \zeta)| d x d y<\infty .
$$

This follows immediately from the fact that $k(z, \zeta)$ is a bounded analytic function of $z$ for each $\zeta$ in $U$ and hence

$$
\begin{aligned}
I & \leqq\|k(z, \zeta)\|_{\infty} \iint_{\Omega}|F(z)| \sum\left|T^{\prime}(z)\right| d x d y \\
& =\|k(z, \zeta)\|_{\infty} \iint_{\Omega}\left|F(z) \lambda^{-1}(z)\right|\left(\sum\left(1-|T z|^{2}\right)\right) d \omega(z) .
\end{aligned}
$$

Now Hölder's inequality, (4.1) and the fact that $F \in H(p, \Gamma)$ yields the desired result.

Note 1 . If $\Gamma$ is finitely generated of the second kind then an analogous 
argument to that in [6] yields

$$
\begin{gathered}
0 \leqq \alpha(\zeta, \zeta)=\iint_{\Omega}|\alpha(z, \zeta)|^{2} d x d y \leqq C\left(1-|\zeta|^{2}\right)^{-2}, \\
|\alpha(z, \zeta)| \leqq C\left(1-|z|^{2}\right)^{-1}\left(1-|\zeta|^{2}\right)^{-1} .
\end{gathered}
$$

Note 2. If one developed an $L_{p}$ theory for automorphic forms of dimension $-2 q, q$ an arbitrary real $(q>1)$, as in Drasin [2], then the above proposition has an analogous statement (viz. Theorem 3 of [2]).

We can now show

Proposition 9. If $\Gamma$ is finitely generated of the second kind then $H(p, \Gamma) \subseteq(H(q, \Gamma)) \quad(1<p \leqq q \leqq \infty)$.

Proof. We first note that it suffices to show

$$
H(p, \Gamma) \subseteq H(\infty, \Gamma), \quad 1<p<\infty,
$$

for then, if $q>p$, it follows that

$$
\begin{aligned}
\iint_{\Omega}\left|F \lambda^{-1}\right|^{\alpha} d \omega(z) & =\iint_{\Omega}\left|F \lambda^{-1}\right|^{q-p}\left|F \lambda^{-1}\right|^{p} d \omega(z) \\
& \leqq\|F\|_{\infty}^{q-p}\|F\|_{p}^{p}<\infty .
\end{aligned}
$$

To see that (5.4) holds we first assume that $1<p \leqq 2$. Then by (5.1) we have for $F \in H(p, \Gamma)$ and $1 / p+1 / p^{\prime}=1$

$$
\begin{aligned}
|F(\zeta)| & =\left|\iint_{\Omega} F(z) \overline{\alpha(z, \zeta)} d x d y\right| \\
& \leqq\left(\iint_{\Omega}\left|F \lambda^{-1}\right|^{p} d \omega(z)\right)^{1 / p}\left(\iint_{\Omega}|\alpha(z, \zeta)|^{p^{\prime}} \lambda^{-p^{\prime}} d \omega(z)\right)^{1 / p^{\prime}} \\
& =\|F\|_{p}\left(\iint_{\Omega}|\alpha(z, \zeta)|^{p^{\prime}-2}|\alpha(z, \zeta)|^{2} \lambda^{-p^{\prime}+2} d x d y\right)^{1 / p^{\prime}} \\
& \leqq\|F\|_{p} C^{2-\left(1 / p^{\prime}\right)}\left(1-|\zeta|^{2}\right)^{-\left(\left(p^{\prime}-2\right) / p^{\prime}\right)}\left(1-|\zeta|^{2}\right)^{2 / p^{\prime}} \\
& =\|F\|_{p} C^{2-\left(1 / p^{\prime}\right)}\left(1-|\zeta|^{2}\right)^{-1}
\end{aligned}
$$

by Hölder's inequality (5.2) and (5.3). This completes the proof in case $1<p \leqq 2$.

Now we note that an analogous argument based upon a similar reproducing formula for $p$-integrable holomorphic automorphic forms $G$ of dimension $-2 q<-2$ (cf. Drasin [2]) would show that such forms are 
bounded if $1<p \leqq 2$, i.e.,

$$
\sup _{z \in U}|G(z)|\left(1-|z|^{2}\right)^{q}<\infty .
$$

Hence, we let $p>2$ and $F \in H(p, \Gamma)$, and choose an integer $m$ such that $m<p \leqq m+1$. It follows immediately that $F^{m}(z)$ is a $p / m$ integrable form of dimension $-2 m$ and since $1<p / m \leqq 2$ the remark above yields that $\left|F^{m}(z)\right|\left(1-|z|^{2}\right)^{m} \leqq C$ for all $z$ in $U$, i.e.

$$
\sup _{z \in U}|F(z)|\left(1-|z|^{2}\right) \leqq C^{1 / m}<\infty .
$$

This completes the proof.

REMARK 1. We note that it is easy to see that if one used an arbitrary system of factors of automorphy $\{\rho(z, T): T \in \Gamma\}$ instead of $T^{\prime}(z)$ then the statements and results of all of the above theorems go through exactly as is.

\section{BIBLIOGRAPHY}

1. A. F. Beardon, The exponent of convergence of Poincaré series, Proc. London Math. Soc. (3) 18 (1968), 461-483. MR 37 \#2986.

2. D. Drasin, Cusp forms and Poincaré series, Amer. J. Math. 90 (1968), 356-365. MR 37 \#5384.

3. P. L. Duren, B. W. Romberg and A. L. Shields, Linear functionals on $H_{p}$ spaces with $0<p<1$, J. Reine Angew. Math. 238 (1969), 32-60. MR 41 \#4217.

4. T. A. Metzger, On integrable and square integrable automorphic forms, Thesis, Purdue University, West Lafayette, Ind., 1971.

5. T. A. Metzger and K. V. Rajeswara Rao, On integrable and bounded automorphic forms. I, Proc. Amer. Math. Soc. 28 (1971), 562-566. MR 43 \#6432.

6. - On integrable and bounded automorphic forms. II, Proc. Amer. Math. Soc. 32 (1972), 201-204.

7. K. V. Rajeswara Rao, Fuchsian groups of convergence type and Poincaré series of dimension -2, J. Math. Mech. 18 (1969), 629-644. MR 38 \#6065.

8. A. Selberg, On discontinuous groups in higher dimensional symmetric spaces, Contributions to Function Theory, Tata Institute of Fundamental Research, Bombay, India., 1960, pp. 147-164. MR 24 \#A188.

9. M. Tsuji, Potential theory in modern function theory, Maruzen, Tokyo, 1959. MR 22 \#5712.

Department of Mathematics, Texas A\&M University, College Station, Texas 77843

Current address: Department of Mathematics, University of Pittsburgh, Pittsburgh, Pennsylvania 15213 